Bio - grafia. Escritos sobre la Biología y su Enseñanza. ISSN 2027

Edición Extraordinaria. p.p. 1170- 1180

Memorias del VIII Encuentro Nacional de Experiencias en Enseñanza de la Biología y la Educación Ambiental. III Congreso Nacional de Investigación en Enseñanza de la Biología.

\title{
IDENTIFICACIÓN DEL CONOCIMIENTO DIDÁCTICO DEL CONTENIDO DE BIOLOGIA DE DOS PROFESORES DE BIOLOGÍA EN EL DISEÑO E IMPLEMENTACIÓN DE TALLERES: DOS ESTUDIOS DE CASO.
}

\section{PEDAGOGICAL CONTENT KNOWLEDGE IDENTIFICATION OF TWO BIOLOGY TEACHERS IN THE DESIGN AND IMPLEMENTATION WORKSHOP: TWO CASE STUDIES.}

\section{B Yessica Alejandra Martínez Sánchez \\ Eliana Gizeth Rojas Beltrán ${ }^{1}$ \\ Yolanda Catalina Vallejo Ovalle ${ }^{2}$}

\section{Resumen}

Esta ponencia presenta los resultados parciales de un estudio que tiene como objetivo caracterizar el conocimiento didáctico del contenido biológico (CDCB) de dos profesores al momento de diseñar y desarrollar talleres de Biología en el aula de clases de una Institución Educativa Distrital de Bogotá (Colombia). El enfoque metodológico fue interpretativo de tipo cualitativo, el método consistió en un estudio de caso a través de técnicas como el análisis de contenido y la entrevista semi-estructurada, análisis documental y la videograbación. Las categorías utilizadas fueron a partir de lo planteado por Valbuena (2007) en relación con el CDCB, y a partir de ello se realizó el análisis de dos categorías, la primera es los contenidos biológicos por enseñar, la segunda es las estrategias metodológicas para la enseñanza de la Biología.

Los profesores tienen un $\mathrm{CDC}$ el cual es importante al momento de diseñar e implementar talleres en el aula de clases, cada profesor desarrolla el taller de formas distintas, sin embargo durante el desarrollo apuntan a que los contenidos sean seleccionados para que sean comprendidos por los estudiantes el profesor 1 desde lo conceptual, el profesor 2 desde los conceptual, procedimental y actitudinal y por otro lado; en la estrategia del taller se hace una organización de esos contenidos en base a unas actividades relacionadas con el contexto de las estudiantes y con las dificultades

\footnotetext{
${ }^{1}$ Estudiantes de Décimo Semestre de Licenciatura en Biología. Universidad Pedagógica Nacional. Correo Electrónico: jams1208@hotmail.com, elgirobe@hotmail.com.

${ }^{2}$ Profesora Departamento de Biología. Universidad Pedagógica Nacional. Correo yvallejo@pedagogica.edu.co
} 
Bio - grafia. Escritos sobre la Biología y su Enseñanza. ISSN 2027

Edición Extraordinaria. p.p. 1170- 1180

Memorias del VIII Encuentro Nacional de Experiencias en Enseñanza de la Biología y la Educación Ambiental. III Congreso Nacional de Investigación en Enseñanza de la Biología.

que presenten las estudiantes; sin embargo no se tienen en cuenta características propias del taller en su desarrollo como la organización de grupos que permite el intercambio de conocimiento entre los estudiantes.

Palabras clave: Conocimiento Didáctico del Contenido Biológico, el taller, enseñanza de la Biología, profesor de Biología.

\begin{abstract}
This paper present the partial results of a study aimed to characterize the didactic knowledge of the biological content (CDCB) two teachers when designing and developing workshops in the classroom of a District Educational Institution of Bogota (Colombia). The interpretative qualitative methodological approach, the method consisted of a case study through techniques such as content analysis and semi-structured interviews, document analysis and videotape. The categories used were from the issues raised by Valbuena (2007) concerning the CDCB, already from this analysis of information units 3 categories was made, the first is the methodological strategies for teaching biology, second is the difficulty of teaching and learning of biology and finally the assessment of the learning process of biology and from this integration CDCB that achieves the teacher to design and implement workshops in the classroom Biology shown classes.
\end{abstract}

Keywords: Teaching Biological Knowledge Content, workshop, teaching biology.

\title{
Introducción
}

El papel del profesor dentro del acto educativo es de gran importancia porque a partir de la propuesta de estrategias de enseñanza, logra dinamizar los contenidos y objetivos de aprendizaje que se van a llevar a cabo, por lo tanto, dentro de la enseñanza de la Biología es importante que aquellas estrategias que proponga el profesor propicien que el estudiante desarrolle capacidades y habilidades para resolver problemas de su realidad, donde la curiosidad y la creatividad jueguen un papel fundamental en la construcción de su conocimiento. 
Bio - grafia. Escritos sobre la Biología y su Enseñanza. ISSN 2027

Edición Extraordinaria. p.p. 1170- 1180

Memorias del VIII Encuentro Nacional de Experiencias en Enseñanza de la Biología y la Educación Ambiental. III Congreso Nacional de Investigación en Enseñanza de la Biología.

En esta perspectiva, en el estudio realizado se pretende indagar acerca del CDCB, del profesor de biología al momento de diseñar e implementar talleres como una estrategia de enseñanza aprendizaje. El conocimiento didáctico del contenido identifica los cuerpos de conocimiento distintivos para la enseñanza. Representa la mezcla entre materia y didáctica por la que se llega a una comprensión de como determinados temas y problemas se organizan, se representan y se adaptan a los diversos intereses y capacidades de los alumnos. (Shulman, 1987). En este sentido Valbuena (2007) plantea que para el profesor es importante en la estructuración que haga de los contenidos de las lecciones, la selección de las estrategias de enseñanza, la comprensión de las dificultades de aprendizaje de los alumnos y el aprovechamiento de sus concepciones.

Es importante indicar que en la investigación se abordará el conocimiento didáctico del contenido biológico $(C D C B)$, éste comprende unos componentes que en el presente estudio serán tomadas en cuenta: Los contenidos biológicos por enseñar, las estrategias metodológicas para la enseñanza de la biología (específicamente del taller), las dificultades en la enseñanza-aprendizaje de la biología, la evaluación de los aprendizajes de la biología. (Valbuena, 2007)

El taller se asumirá desde Reyes (1989) como una realidad integradora, compleja, reflexiva, en que se unen la teoría y la práctica como fuerza motriz del proceso pedagógico, orientado a una comunicación constante con la realidad social y como un equipo de trabajo altamente dialógico formado por docentes y estudiantes.

Este trabajo esta direccionado a la problematización del CDC que configura el profesor de Biología al diseñar e implementar talleres para la enseñanza de la Biología, este interés surgió a partir de la realización de la práctica pedagógica ${ }^{2}$ se pudo hallar una cuestión que se encontraba inmersa en las aulas de clases, en relación a los procesos de transformación didáctica que realizaban los profesores al pensar los talleres para la enseñanza de biología, de tal modo que tenían como base libros de texto o internet, que se estructuraban con preguntas cerradas de un texto base. Por lo tanto, el taller a ser tan utilizado frecuentemente en la enseñanza de la Biología

\footnotetext{
${ }^{2}$ La Práctica Pedagógica en la UPN, se ubica institucionalmente en el tercer campo de estudio del área de Formación Pedagógica y Didáctica, cuyo propósito es " la formación de un maestro caracterizado por ser intelectual de la pedagogía, un trabajador de la cultura y un agente de cambio social, con una misión definida: ser transformador de las organizaciones sociales educativas, empleando como herramientas la producción de teorías pedagógicas, la transformación de las prácticas pedagógicas e incidir en las políticas educativas." 2
} 
Bio - grafia. Escritos sobre la Biología y su Enseñanza. ISSN 2027

Edición Extraordinaria. p.p. $1170-1180$

Memorias del VIII Encuentro Nacional de Experiencias en Enseñanza de la Biología y la Educación Ambiental. III Congreso Nacional de Investigación en Enseñanza de la Biología.

se hace necesario indagar sobre el CDC al momento de ser diseñados e implementados por los profesores en el aula de clases.

De acuerdo con lo propuesto anteriormente, se identificó una problemática que giraba en torno a la caracterización del CDC de dos profesores de Biología y como incide este conocimiento al momento del diseño e implementación de talleres. A partir lo anterior, para esta ponencia se presentaran una aproximación a dos categorías del $C D C B$, aclarando que no se pretende limitar el $C D C B$ de los docentes a estos dos componentes.

\section{Metodología}

Esta investigación se realiza desde el paradigma interpretativo donde Ramírez, L., et. al (2004) indica que existen múltiples realidades construidas por el sujeto y este a su vez se configura en diversos significados de acuerdo a las situaciones en las que se encuentran, la investigación tendrá una postura cualitativa porque según Cerda (2008) centra su análisis en la descripción del fenómeno social y del fenómeno observado.

El estudio se desarrolló a partir de dos estudios de caso de dos profesores de básica secundaria de una Institución Educativa Distrital de Bogotá (Colombia), con los cuales se identificara: el significado que le dan al taller como una estrategia de enseñanza-aprendizaje y los contenidos biológicos que allí presentan. Teniendo en cuenta lo anterior, el estudio de caso según Stake (2005) nos permite "estudiar la particularidad y la complejidad de un caso singular o específico no general, para llegar a comprender su actividad en circunstancias importantes".

Este estudio se desarrolló en varias fases, la construcción de la problemática y revisión de antecedentes y referentes con el propósito de tener unos fundamentos teóricos (Conocimiento Didáctico del contenido biológico $C D C B$, documentos institucionales), el diseño de técnicas para recolectar la información (entrevistas talleres y video grabación), sistematización y análisis de contenido que en esta investigación será crucial debido a que conjuga un conjunto de procesos interpretativos del productos comunicativos (mensajes, textos o discursos) que proceden de procesos singulares (en este caso de los profesores) de comunicación previamente registrados (Piñuel, J. 2002). Por último se hará una socialización de los resultados.

Como se indicó anteriormente el análisis del contenido será lo fundamental en la investigación y ésta técnica se utilizara alrededor de unas categorías y de unas unidades de información extraídas en cada instrumento. Estas son: Contenidos Biológicos por enseñar ( $A)$, Las estrategias metodológicas para la enseñanza de la Biología (B). Características de los estudiantes (C), 
Bio - grafia. Escritos sobre la Biología y su Enseñanza. ISSN 2027

Edición Extraordinaria. p.p. 1170- 1180

Memorias del VIII Encuentro Nacional de Experiencias en Enseñanza de la Biología y la Educación Ambiental. III Congreso Nacional de Investigación en Enseñanza de la Biología.

Dificultades en la enseñanza-aprendizaje de la Biología (E), Evaluación del proceso de aprendizaje de la Biología (F). En este caso, solo se presentaran aproximaciones de análisis a la primera y segunda categoría.

\section{Resultados y discusión}

A continuación se presentan las aproximaciones al análisis de las categorías contenidos biológicos por enseñar y las estrategias metodológicas desde tres fuentes: la entrevista, el taller y la video grabación; con el fin de contrastar lo pensado, planeado e implementado. Para efectos de organización, la codificación está organizada de la siguiente manera: A y B, representan las categorías, los números representan la unidad de información, P1 O P2 son los casos y las palabras EN (entrevista), TA (taller), VI (videograbación).

Los contenidos biológicos por enseñar ( $A$ ): Los hallazgos desde las fuentes del taller y videograbación son escasos, donde mencionen específicamente los contenidos biológicos a enseñar, por lo tanto, se realiza el análisis de acuerdo a las unidades de información encontradas como se muestra a continuación.

\section{PROFESOR 1}

- Teniendo en cuenta los contenidos biológicos por enseñar en el taller, es necesario mencionar que la temática central es la reproducción celular, sin embargo presenta definiciones cortas de los temas términos mostrados en la unidad de información. Con ello se podría decir que el profesor 1 privilegia contenidos de tipo conceptual que de acuerdo a Coll, C., et al. (1992), constituye la información que le da sentido a los hechos; dejando un poco de lado lo procedimental y actitudinal.

"La mayoría de células procariotas se reproducen por un proceso llamado fisión binaria. Este es un tipo de proceso de clonación en la que dos células idénticas se derivan de una sola célula." (A-3-P1-

TA)

> En este sentido, esos hechos se ven reflejados desde la videograbación en la ejecución del taller, donde no solo se evidencia que relaciona eventos climáticos cotidianos con la temática expuesta, sino también que propone un proceso procedimental para ejecutarlos, en este último 
Bio - grafia. Escritos sobre la Biología y su Enseñanza. ISSN 2027

Edición Extraordinaria. p.p. 1170- 1180

Memorias del VIII Encuentro Nacional de Experiencias en Enseñanza de la Biología y la Educación Ambiental. III Congreso Nacional de Investigación en Enseñanza de la Biología.

aspecto aplica gráficas y textos científicos, dibujos, esquemas; como se presenta en la siguiente unidad de información.

"Primer punto, esta cara vale 3 (la señala, voltea la hoja) esta cara vale 3, si contesta la otra gráfica, este primer punto se contesta aquí (señala el lugar en la hoja donde se debe contestar), primer punto hay están las claves, hay están las claves, segundo, lee mentalmente el siguiente texto científico, ese texto es el mismo acerca de las nubes (señala hacia arriba) de los rayos, si, es el mismo pero entonces ya no le va a decir que es la nubecita, que cayó un rayo o descarga eléctrica ahi le va a hablar de potentes flujos energéticos extrasolares (se remite a la hoja) entonces haga la lectura y hay unos términos subrayados" (A-1-P1-VI)

\section{PROFESOR 2 (P2)}

> En el taller analizado se encontró que el profesor trabaja la temática de la organización del ser vivo, desde el componente celular y organismico. En este sentido hace una selección de los contenidos que se verán en la clase planteando desde el inicio un objetivo que relaciona los contenidos conceptuales porque tiene en cuenta unos conceptos a desarrollar, procedimental porque enmarca unas actividades dirigidas y actitudinal porque pretende que las estudiantes valoren la importancia del tema, como se muestra en la siguiente unidad de información

"Reconocer las partes de la célula y su morfo fisiología, valorando la importancia de ésta como unidad funcional de los seres vivos, sustentando sus conocimientos, elaborando redes conceptuales coherentes que representa en forma oral o escrita".(A-1-P2-TA)

- Como indica Coll, C., et al (1992). Los contenidos procedimentales corresponden a actividades escolares para el aprendizaje y la enseñanza, engloba hábitos, técnicas, algoritmos, habilidades, estrategias, métodos y rutinas diversas para para llegar a las metas; probablemente esto es lo que el profesor 2 quiere realizar con la práctica de proyectos ambientales y calendarios ecológicos.

"De los seres vivos hablamos de proyectos de basura 0 , del de tenencia responsable de animales. Del calendario ecológico, ósea del componente ecológico, hemos visto: el día sin carro, la semana santa donde hablamos del loro oreji amarillo y de la palma de cera, del día del agua del día del medio ambiente y una reflexión anti taurina". (A-3-P2-VI) 
Bio - grafia. Escritos sobre la Biología y su Enseñanza. ISSN 2027

Edición Extraordinaria. p.p. 1170- 1180

Memorias del VIII Encuentro Nacional de Experiencias en Enseñanza de la Biología y la Educación Ambiental. III Congreso Nacional de Investigación en Enseñanza de la Biología.

Las estrategias metodológicas para la enseñanza de la biología (B): Los resultados presentados para esta categoría fueron obtenidos a través de las fuentes de información como la entrevista, el taller y la videograbación.

PROFESOR 1 (P1)

$>$ El taller es visto por el profesor como la acumulación de estrategias que pueden ser como los mapas conceptuales, resúmenes y el parafraseo de conceptos, cuando según Egg (1999) los talleres son comprendidos como una estrategia de enseñanza/aprendizaje, donde el profesor orienta unos conocimientos y ayuda al estudiante en su trabajo de" aprender a aprender" por medio de actividades planeadas, articuladas y sistematizadas hacia la realización de algo, esta actividad consiste en "aprender haciendo" interactuando con los otros por medio de un trabajo cooperativo. Lo dicho por el caso se presenta a continuación:

"Un taller es, yo lo podría tomar como una suma de varias estrategias entre ellas el mapa conceptual...un resumen, incluso hasta el parafraseo, sí que es la repetición cíclica de los conceptos" (B-1-P1-EN)

> En las unidades presentadas se halla una relación en el diseño del taller en cuanto a las actividades enfocadas a la resolución de preguntas, al parafraseo, a las representaciones y esquemas, de tal manera que en el taller de biología se podría deducir que es de tipo descriptivo, ya que para resolver las preguntas planteadas las estudiantes deben basarse de las definiciones específicas que propone el profesor 1 en el taller.

"¿Qué requiere la célula para dividir el núcleo?"(B-2-P1-TA)

"Escribe las 3 partes fundamentales de procariotas:"(B-3-P1-TA)

"Haz un esquema:"(B-4-P1-TA)

"Realiza un proceso de ciclo celular y mitosis de una célula somática con 14 cromosomas (Centeno)."(B-10-P1-TA)

> El profesor dentro del taller tiene en cuenta la organización de sus estudiantes en el aula de clase, ya sea por sus nombres en orden alfabético o por las dificultades que presentan las estudiantes en su dinámica de aprendizaje. 
Bio - grafia. Escritos sobre la Biología y su Enseñanza. ISSN 2027

Edición Extraordinaria. p.p. 1170- 1180

Memorias del VIII Encuentro Nacional de Experiencias en Enseñanza de la Biología y la Educación Ambiental. III Congreso Nacional de Investigación en Enseñanza de la Biología.

"Ellas están organizadas por orden alfabético, los casos más sensibles de dificultades en hábitos de estudio de pronto si son reacomodadas" (B-3-P1-EN)

"Las niñas de la esquina por favor sepárense, Jenny sepárese, Laura, mejor dicho Valeri y tu (señala la estudiante) pásense una en la mesa del lado y la otra en la mesa gris con el cuaderno y...."

(B-3-P1-VI)

PROFESOR 2 (P2)

> En el caso del profesor 2 el taller se vislumbra como una actividad, sin embargo a esta actividad le atribuye una organización, unos fines y un procedimiento para orientar el proceso; sin embargo Sosa (2002) indica que dentro de la estructura del taller debe haber una Distribución de grupos, teniendo en cuenta que en el taller el saber se construye no solo haciendo; sino en grupo, es decir de manera cooperativa, es necesario organizar a los participantes en grupos facilitándoles la oportunidad de relacionarse con los demás compartiendo $e$ intercambiando habilidades, conocimientos e intereses, lo dicho por el caso se presenta a continuación:

"Un taller es un escrito el cual tiene unos aspectos tales como una conducta de entrada, unos objetivos, unos materiales, un procedimiento, unas conclusiones y desde luego la bibliografía" (B-1P2-EN)

- El profesor 2 es variable en cuanto al desarrollo de sus talleres. Los talleres se engloban desde la dinámica de responder preguntas basadas en un texto, hasta la representación por medio de diversos dibujos sobre videos, anuncios o textos. Sobre este último elemento es importante citar a como indica Nauman, B. (1991) dibujar es el equivalente a pensar, el dibujo constituye la escritura del artista, su opinión, su forma de ver las cosas.

\section{Actividades}

1. Taller de ideas previas .Consulta.

2. La célula de Juan -LECTURA

3. Dictado de un dibujo.

4. Elaboración y revisión de la célula.

5. Bio letras

6. Observación microscópica.
Taller de ideas previas

Recordar/consultar

1. Definir de célula.

2. Realizar resumen de la Historia y la Teoría Celular (usando material de apoyo adjunto: Unidad de vida y Teoría celular). 
Bio - grafia. Escritos sobre la Biología y su Enseñanza. ISSN 2027

Edición Extraordinaria. p.p. 1170- 1180

Memorias del VIII Encuentro Nacional de Experiencias en Enseñanza de la Biología y la Educación Ambiental. III Congreso Nacional de Investigación en Enseñanza de la Biología.

\begin{tabular}{|c|c|}
\hline $\begin{array}{c}\text { 7. Evaluación de partes de célula } \\
(B-2-P 2-T A)\end{array}$ & $\begin{array}{c}\text { 3. Partes de la célula y funciones de estas. } \\
\text { Realizar cuadro. } \\
\text { 4. Hacer esquemas de Célula Vegetal y } \\
\text { Animal. } \\
(B-3-P 2-T A)\end{array}$ \\
\hline
\end{tabular}

> El profesor de acuerdo a lo evidenciado realiza talleres como medio para motivar a las estudiantes sobre los hechos actuales de la biología (conocimiento contextual y de la disciplina) y ampliar sus saberes y por otro lado, enfatizar las competencias lectoras mediante libros de superación y crecimiento personal, por medio de actividades de su gusto como la expresión oral. De acuerdo a Shulman (1987) el conocimiento didáctico del contenido representa la mezcla entre materia y didáctica por la que se llega a una comprensión de como esos temas y problemas se organizan y se adaptan a los diversos intereses y capacidades de los alumnos.

"Traigo 40 periódicos, los reparto en clase con temas afines a biología y las niñas en una hojita deben hacer su resumen como quieran, las niñas entonces quieren eso y la expresión oral". (B-2-

P2-EN)

\section{Conclusiones}

- Se identificó que el CDCB de los estudios de caso se lleva al aula de manera distinta cuando va a desarrollar talleres en su clase, tanto de los contenidos que selecciona y a los cuales le da prioridad, hasta la organización de las actividades que realiza dentro del taller.

- Los contenidos del profesor 1 son seleccionados de acuerdo al tema en particular que va a enseñar y le da prioridad a los contenidos de tipo conceptual.

- El profesor 2 tiene en cuenta en la selección de sus contenidos, lo conceptual, procedimental y actitudinal de ellos.

- Dentro de su CDCB, se identificó que el taller no es concebido como una estrategia por parte de ambos casos sino como un cumulo de actividades, emprendidas para enseñar un concepto biológico, por lo que muchas de sus características no son claras en el desarrollo 
Bio - grafia. Escritos sobre la Biología y su Enseñanza. ISSN 2027

Edición Extraordinaria. p.p. 1170- 1180

Memorias del VIII Encuentro Nacional de Experiencias en Enseñanza de la Biología y la Educación Ambiental. III Congreso Nacional de Investigación en Enseñanza de la Biología.

de ambos talleres, como la organización en grupos que se realiza con el fin de darle la oportunidad a los estudiantes de relacionarse con los demás compartiendo e intercambiando habilidades, conocimientos e intereses.

\section{Bibliografía}

> Cerda, H. (2008). Los elementos de la investigación. como reconocerlos, diseñarlos y construirlos. editorial el buho Ltda.

Egg, E. (1999) EL TALLER UNA ALTERNATIVA DE RENOVACIÓN PEDAGÓgICA. Ed Magisterio. Rio de la Plata, Argentina. Extraído el 23 de agosto del 2013 de la pág. web http://librosgratis.net/book/el-taller-una-alternativa-de-renovacion-pedagogica_81330.html

> Piñuel, j. (2002). Epistemología, metodología y técnicas del análisis de contenido. Universidad Complutense de Madrid. Pág. 1. Extraído el 3 de febrero del 2015 de https://www.ucm.es/data/cont/docs/268-2013-07-29-

Pinuel Raigada AnalisisContenido_2002_EstudiosSociolinguisticaUVigo.pdf

Ramírez, L., Arcilla, A., Buritica, L., Castrillón, J. (2004). PARADIGMAS Y MODELOS DE INVESTIGACION. Fundación Universitaria Luis Amigó. Extraído el 3 de febrero del 2015 de http://virtual.funlam.edu.co/repositorio/sites/default/files/repositorioarchivos/2011/02/0008p aradigmasymodelos.771.pdf

Reyes, M. (1989). EL TALLER EN TRABAJO SOCIAL. Ed. Humanitas. Argentina, Buenos aires. Extraído el 7 de Noviembre del 2013 de la pág. webhttp://aprendeenlinea.udea.edu.co/revistas/index.php/ceo/article/viewFile/1650/1302 UniversityPress. Cita extraída de tesis Doctoral de López, M. (2009).

Sosa G. Mercedes (2002) El taller estrategia educativa para el aprendizaje significativo, Bogotá. Circulo de lectura Alternativa.

Shulman, L. (1987). Knowledge and teaching: foundations of the new reform. Harvard Educational Review, 57(1), 1-22. Traducción castellana (2005): Conocimiento y enseñanza: 
Bio - grafia. Escritos sobre la Biología y su Enseñanza. ISSN 2027

Edición Extraordinaria. p.p. 1170- 1180

Memorias del VIII Encuentro Nacional de Experiencias en Enseñanza de la Biología y la Educación Ambiental. III Congreso Nacional de Investigación en Enseñanza de la Biología.

fundamento de la nueva reforma. Profesorado. Revista de Currículum y Formación de Profesorado, 9(2). Extraído el 4 de julio del 2015 de http://www.ugr.es/ recfpro/rev92ART1.pdf.

> Valbuena, E. (2007).EL CONOCIMIENTO DIDÁCTICO DEL CONTENIDO BIOLÓGICO: ESTUDIO DE LAS CONCEPCIONES DISCIPLINARES Y DIDÁCTICAS DE FUTUROS DOCENTES DE LA UNIVERSIDAD PEDAGÓGICA NACIONAL. Extraído el 1 de Marzo de 2014 de la página web http://eprints.ucm.es/7731/1/T30032.pdf

Stake, R. (2005). Investigación con estudio de casos. Madrid, Morata. Extraído el 3 de febrero del 2015

de http://investigacionsocial.sociales.uba.ar/files/2013/03/STAKE_investigacion-conestudio-decasos.pdf. 